\title{
Renal resistive index in mouse model
}

\author{
Zejun Ma', Shan Lu², Lexi Ding ${ }^{3}$ and Qiulun $\mathrm{Lu}^{4 *}$ \\ ${ }^{1}$ Key Laboratory of Hormones and Development (Ministry of Health), Tianjin Medical University, China \\ ${ }^{2}$ Department of Pharmacology, University of California, USA
}

${ }^{3}$ Department of Ophthalmology, Xiangya Hospital, Center South University, China

${ }^{4}$ Key Laboratory of Molecular Biophysics of the Ministry of Education, Huazhong University of Science and Technology, China

*Corresponding author: Qiulun Lu, Key Laboratory of Molecular Biophysics of the Ministry of Education, College of Life Science and Technology and Center for Human Genome Research, Huazhong University of Science and Technology, Wuhan, China

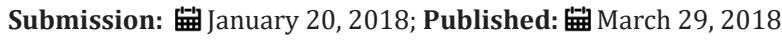

\begin{abstract}
Renal Resistive Index (RI) analysis by Doppler ultrasonography has been shown to be associated with renal injury in clinics. Additionally, renal RI was applied on animals including rabbit, rat, and mouse in researches, which shows renal RI could also reflect the degree of renal impairment. There are two different methods for renal RI analysis in mouse model currently. One of them is widely performed as intrarenal RI, while the other is suprarenal RI. In this review, the difference of these two methods will discussed in depth, and their advantages and disadvantages will be described.
\end{abstract}

Keyword: Ultrasonography; Intrarenal RI; Suprarenal RI; Renal injury

\section{Review}

Over decades, ultrasonography, reflecting the changes of renal function and morphology, has been used in the assessment of chronic kidney disease [1,2]. Besides detection of renal macroscopic vascular abnormalities, ultrasonography could identify changes in blood flow at the microvascular level $[3,4$,$] . In$ clinical application, evaluation of vascular impedance at different sites of the renal parenchyma may indicate functional or structural alterations within the kidneys, which provides useful diagnostic and prognostic information [5]. Based on the Doppler ultrasonography, the Resistive Index (RI), as a novel parameter calculated from the blood flow velocities in vessels among the cardiac cycle, is used as pulse-wave Doppler (PW-Mode) measurement of resistance and compliance in downstream arteries [6]. Differences in RI reflect downstream vasculature resistance, which could at least in part depend on the degree of vascular compliance [7]. However, as compliance increased, it became increasingly less dependent on resistance. Despite of that, the higher index of RI, the higher is velocity difference between the systole and the diastole [3].

In 1986, Rigsby et used RI values to evaluate acute rejection after renal allografts [8]. In the following years, RI was considered a reliable and repeatable renal function parameter in a variety of clinical settings, such as detection and management of renal artery stenosis [9], evaluation of progression risk in Chronic Kidney Disease (CKD) [10], and differential diagnosis in acute and chronic obstructive renal disease. Additionally, elevated Renal RI has been shown to correlate with the degree of renal injury in hypertensive patients [11]. Surprisingly, among those hypertensive patients, RI analysis can also be considered as a complement predictor of cardiovascular and renal outcomes [11].
Due to the repeatable and reliable RI analysis in clinics, renal RI is gradually accepted in research studies with different animal models. For example, RI could be useful in predicting the course of acute renal failure in rabbits [12]; In rats, renal RI increased significantly in the anheptic stage and decreased drastically after the reperfusion in liver transplantation model [13]. Additionally, there are publications reported that renal RI is associated with renal impairment in mouse model. Westergren et al. [14] found the increased renal RI in ob/ob mouse, the obese type 2 diabetic leptindeficient mouse, accompanying with the renal injury. Xu et al. [15] also demonstrated that high fat diet induced the increase of renal RI in mouse mode, with the increased ACR levels.

Renal RI is determined as following:

$\mathrm{RI}=(\mathrm{PSV}-\mathrm{EDV}) / \mathrm{PSV}$

PSV is peak systolic velocity, and EDV is End-Diastolic Velocity. The blood flow is susceptible to instabilities of the environment and operation procedure. There are multiple variables that affect the blood flow involved in ultrasound measurement, such as temperature, humidity, and et al. Also, blood flow could be affected by the mouse status, including body temperature and the degree of anesthesia. As what we concern, the method itself of blood flow velocity measurement is not precise and reliable. It could be affected by the position of mouse, the angles for blood flow measurement, the site for measurement, and et al. Since the RI value is the ratio of velocities, instead of absolute value, it is stable and unbiased parameters for flow analysis.

Up till now, it was reported that there are two different types of renal RI, known as intra renal RI and suprarenal RI. Among those two 
available index, intra renal RI is widely accepted and used (Figure 1) [14]. The intrarenal RI is based on standard B mode examination of the kidney to identify targeted intrarenal artery. This central segmental artery, is identified with color-Doppler images of the renal vascular tree. Subsequently, renal flow velocity was measured using PW Doppler. On the other hand, suprarenal RI was performed and described by Xu et al. [15]. After the probe was placed on the mouse abdomen, transverse image of the suprarenal artery at the level of the suprarenal gland were obtained. Then, the probe was switched to the parasternal position to capture longitudinal images of the abdominal aorta and left renal artery. Later, the suprarenal artery was confirmed by the color Doppler. Once reaching the right position, images of blood flow at the entrance of suprarenal artery were taken via PW Doppler (Figure 2). Compare to intra renal, suprarenal RI is more repeatable and comparable, as the site of measurement is consistent. To be more specific, the entrance of suprarenal artery is easier to find and appears to be consistent in different mice, whereas intra renal RI has no solid standard for identification. Moreover, Xu et al. [15] also demonstrated that suprarenal RI is found to be associated with renal injury in high fat diet-fed mice. In his study, suprarenal RI is positive correlated with GFR at the early stage of high fat diet-induced renal injury. Even though there are multiple advantages for suprarenal RI, it is still necessary to include more experiments to validate its efficacy and accuracy. Furthermore, whether suprarenal RI is also suitable for rat and other animals are still understudied.

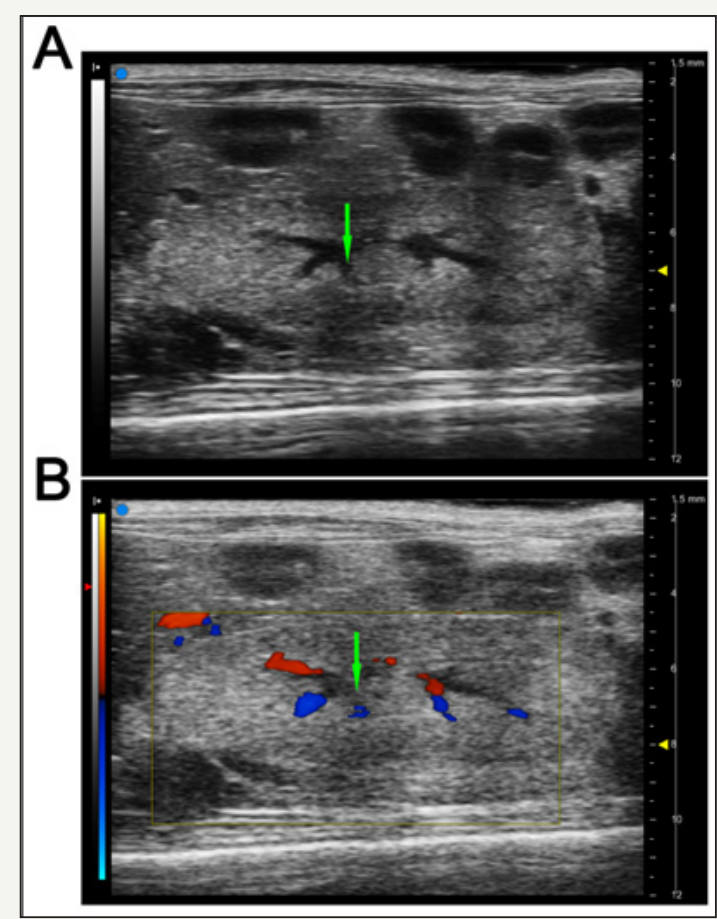

Figure 1 : Representative images for measurement of intrarenal flow.

A: Typical image of kidney tissue was performed using $B$ mode. The intrarenal artery was marked in Green as segmental intrarenal arteries.

B: The representative images of color Doppler was performed to confirm the intrarenal arteries.

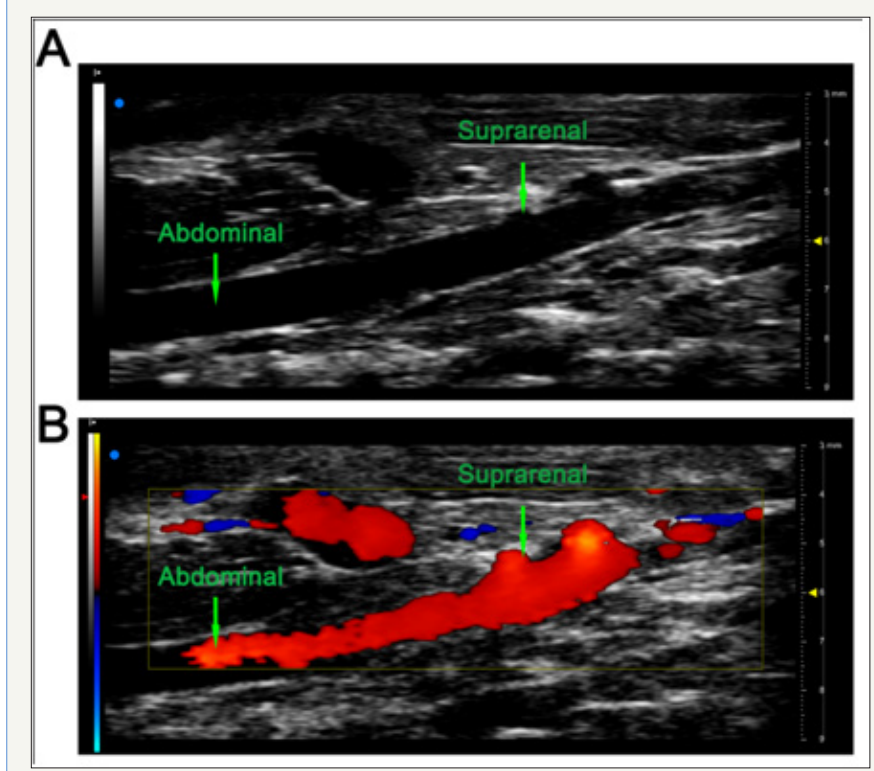

Figure 2 : Representative images for measurement of suprarenal flow.

A: Typical image of abdominal artery was performed using $B$ mode. The suprarenal artery and abdominal artery were marked in Green as segmental intrarenal arteries.

$\mathrm{B}$ : The representative images of color Doppler was performed to confirm the entrance of suprarenal artery.

\section{Acknowledgment}

This study was supported by the grant from the National Natural Science Foundation of China (81500720) and the grant from Health Bureau Technology Fund of Tianjin (2014KZ092).

\section{References}

1. Tublin ME, Bude RO, Platt JF (2003) The resistive index in renal Doppler sonography: where do we stand? American journal of roentgenology 180(4): 885-892.

2. Shakourirad A, Ataeefar M, Jozaghi S (2009) Assessment of the effect of radio contrast media on resistive index of renal artery by color doppler sonography. Saudi journal of kidney diseases and transplantation: an official publication of the Saudi Center for Organ Transplantation, Saudi Arabia 20: 91-6.

3. H Hosojima (2002) Intrarenal resistive index (RI) from renal duplex Doppler sonography in type II diabetic patients with nephropathy. Nihon rinsho. Japanese journal of clinical medicine 60 Suppl (8): 676-681.

4. Platt JF, Ellis JH, Rubin JM, DiPietro MA, Sedman AB (1990) Intra renal arterial Doppler sonography in patients with nonobstructive renal disease: correlation of resistive index with biopsy findings. AJR American journal of Roentgenology 154(6): 1223-1227.

5. Kim ES, Kim HJ, Kim YJ, Lee SM, Lee HJ, et al. (2013) Resistive index as a predictor of acute kidney injury caused by an angiotensin converting enzyme inhibitor or angiotensin II receptor blocker in chronic kidney disease patients. Kidney Res Clin Pract 32(4): 158-163.

6. Hosojima H (1998) Intrarenal Resistive Index (RI) from renal duplex Doppler sonography in diabetic patients with nephropathy. Nihon rinsho Japanese journal of clinical medicine 56Suppl (3): 505-509.

7. Krumme B, Hollenbeck M (2007) Doppler sonography in renal artery stenosis--does the Resistive Index predict the success of intervention?. Nephrology dialysis transplantation 22(3): 692-696. 
8. Wan SK, Ferguson CJ, Cochlin DL, Evans C, Griffiths DF (1989) Duplex Doppler ultrasound in the diagnosis of acute renal allograft rejection. Clin Radiol 40(6): 573-576.

9. Radermacher J, A Chavan, J Bleck, A Vitzthum, B Stoess, et al. (2001) Use of Doppler ultrasonography to predict the outcome of therapy for renalartery stenosis. N Engl J Med 344(6): 410-417.

10. Radermacher J, Ellis S, HallerRenal H (2002) resistance index and progression of renal disease. Hypertension 39(2pt 2): 699-703.

11. Doi Y, Iwashima Y, Yoshihara F, Kamide K, Hayashi S, et al. (2012) Renal resistive index and cardiovascular and renal outcomes in essential hypertension. Hypertension 60(3): 770-777.

12. Yoon DY, Kim SH, Kim HD, Na DG, Goo JM, et al. (1995) Doppler sonography in experimentally induced acute renal failure in rabbits.
Resistive index versus serum creatinine levels. Investigative radiology 30: $168-172$.

13. Kong H Y, Chen F, He Y, Wu LJ, Wang LQ et al. (2013) Intrarenal resistance index for the assessment of acute renal injury in a rat liver transplantation model. BMC nephrology 14: 55.

14. Westergren HU, Gronros J, Heinonen SE, Miliotis T, Jennbacken K, et al. (2015) Impaired Coronary and Renal Vascular Function in Spontaneously Type 2 Diabetic Leptin-Deficient Mice. PloS one 10(6): e0130648.

15. Xu H, Ma Z, Lu S, Li R, Lyu L, et al. (2017) Renal Resistive Index as a Novel Indicator for Renal Complications in High-Fat Diet-Fed Mice. Kidney Blood Press Res 42(6): 1128-1140.
Creative Commons Attribution 4.0

International License

For possible submissions Click Here
Submit Article

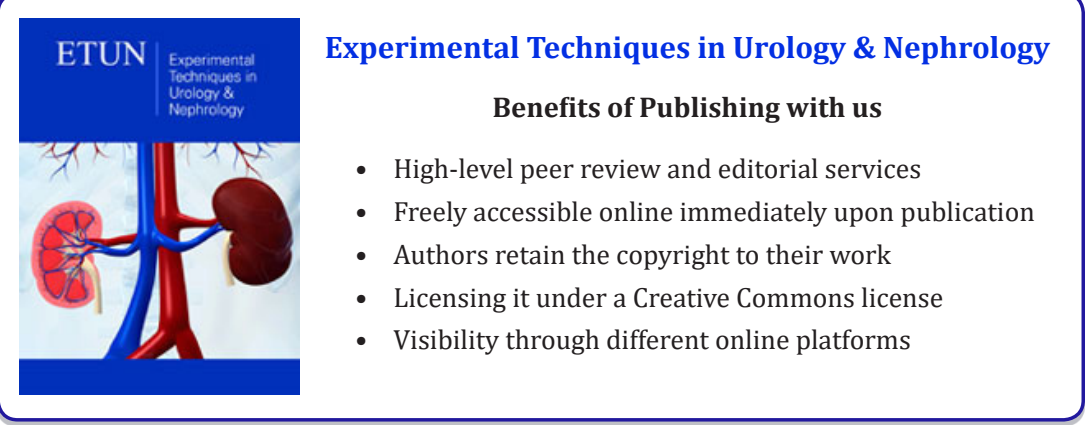

